\title{
New inhibitors of DNA methyltransferase 1 based on cytosine scaffold
}

\author{
Agnieszka Fedoruk-Wyszomirska, WoJcIech T. MarkieWICZ, JAN BARCisZEWSKI * \\ Institute of Bioorganic Chemistry, Polish Academy of Sciences, Poznań, Poland
}

\section{United States Patent}

Patent number: US9040490 B2

Application number: US 13/576709

Cytosine analogue, a method of preparation of a cytosine analogue, a DNA methyltransferase 1 inhibitor, a method for DNA methylation inhibition, the use of the analogue in the treatment of diseases associated with deviations from normal DNA methylation

Inventors: J. Barciszewski, W.T. Markiewicz, E. Adamska, B. Plitta, M. Giel-Pietraszuk, E. Wyszko, M. Markiewicz, A. Fedoruk-Wyszomirska, T. Kulinski, M. Chmielewski

Assignee: Institute of Bioorganic Chemistry, PAS, Poznań

Date of Patent: 26. 05. 2015

\section{European Patent Application}

PCT number: PCT/PL2011/000032

Cytosine analogue, a method of preparation of a cytosine analogue, a DNA methyltransferase 1 inhibitor, a method for DNA methylation inhibition, the use of the analogue in the treatment of diseases associated with deviations from normal DNA methylation

Inventors: J. Barciszewski, W.T. Markiewicz, E. Adamska, B. Plitta, M. Giel-Pietraszuk, E. Wyszko, M. Markiewicz, A. Fedoruk-Wyszomirska, T. Kulinski, M. Chmielewski

Assignee: Institute of Bioorganic Chemistry, PAS, Poznań

Application Date: 17. 03. 2011

\section{Polish Patent Application}

Polish Application number: P 390769

Cytosine analogue, a method of preparation of a cytosine analogue, a DNA methyltransferase 1 inhibitor, a method for DNA methylation inhibition, the use of the analogue in the treatment of diseases associated with deviations from normal DNA methylation

Inventors: J. Barciszewski, W.T. Markiewicz, E. Adamska, B. Plitta, M. Giel-Pietraszuk, E. Wyszko, M. Markiewicz, A. Fedoruk-Wyszomirska, T. Kulinski, M. Chmielewski

Assignee: Institute of Bioorganic Chemistry, PAS, Poznań

Application Data: 19. 03. 2010

Published also as: EP2556081A2, US20120322755, WO2011115513A2, WO2011115513A3, WO2011115513A9

\begin{abstract}
In this study, a cytosine analog and its preparation method and a DNA methyltransferase 1 inhibitor and a method for DNA methylation inhibition are provided for the treatment of diseases associated with deviations from normal DNA methylation. The analog of cytosine comprises of 1, N4, 5, and 6-substituted derivatives of cytosine or 5,6-dihydrocytosine; the analog can be described with a chemical formula where R1 is H, R3, R4, 2-deoxyribosyl, R4 is alkyl or aryl, $\mathrm{X}$ is $\mathrm{N}$ or $\mathrm{C}$ - if $\mathrm{X}$ in formula I is $\mathrm{N}$, then $\mathrm{R} 5$ is no substituent and if $\mathrm{X}$ in formula I and/or II is $\mathrm{C}$ or if $\mathrm{X}$ in formula II is $\mathrm{N}$, then R5 and R6 are independently alkyl, aryl, hydroxyalkyl, aminoalkyl, hydroxyl, carboxyl, amino group, alkoxyl, aryloxyl, aminoalkyl, aminoaryl, thio group, sulfonyl, sulfinyl, or halogen.
\end{abstract}

\footnotetext{
* Corresponding author: Institute of Bioorganic Chemistry, Polish Academy of Sciences, Noskowskiego 12/14, 61-704 Poznań, Poland; e-mail: jan.barciszewski@ibch.poznan.pl
} 


\section{State of the art}

This invention provides a cytosine analog and its preparation method and a DNA methyltransferase 1 inhibitor and a method for DNA methylation inhibition, and the use of this analog in the treatment of diseases associated with deviations from normal DNA methylation. More precisely, the invention relates to various derivatives of cytosine, as well as methods of preparation of mono- and multi-1, 4, 5, and 6-substituted cytosines. In general, the solution relates to providing effective modulators of DNA methylation that could be used in prevention and treatment of diseases associated with DNA methylation level disorders.

The gene expression and genomic stability are controlled thus it is an epigenetic mechanism. One of the main element of this mechanism is the methylation pattern that is specific to the particular organism, which is determined at embryonic stage and invariable throughout the lifetime. Exact reproduction of this pattern in successive cellular divisions is the condition of proper development and function of the organism. Variations in the methylation pattern (epimutations), in which may be increase or decrease in the methylation level, have been described in many cancers. Reversibility of epimutations makes them an attractive therapeutic target in the treatment of cancer and in contrast to genetic mutations, epimutations must be actively maintained by DNA methyltransferase 1 (DNMT1) after successive cell divisions. Methylation inhibitors do not lead to immediate cell death, but they stimulate their proliferation and activation of genes that had been silenced by methylation. However, reactivation of proapoptotic genes and cell cycle regulators by means of demethylation results in apoptosis of tumor cells.

Analysis of variations in genomic DNA methylation pattern as well as correlation of this phenomenon with carcinogenesis have led to the development of a novel approach to cancer treatment and diagnostics.

In mammalian cells, four independently coded methyltransferases are involved in the DNA methylation: DNMT1 (reproducing the methylation pattern in replication process), DNMT3a, DNMT3b, and DNMT3L (responsible for de novo methylation at early stages of embryonal development).

Till date several methyltransferase inhibitors have been identified that can be divided into four groups. The first one includes cytidine analogs like 5-azacytidine, 5-aza-2-deoxycytidine, and zebularine. After phosphorylation, they are incorporated into a newly synthesized DNA at cytosine sites. The lack of the proton at position 5 of these cytosine derivatives stabilizes the covalent bond between DNA and enzyme. Thus, the trapped DNMT1 molecules are excluded from the pool of active enzymes, and the formation of these covalent proteinDNA complexes is considered as a reason of the high toxicity of those inhibitors. This leads to a decrease in the level of methylation of replicated DNA. It has been suggested that 5 -azacytidine affects other cellular processes also via altering expression of genes that were not regulated by DNA methylation prior to the treatment. Other group of DNMT1 inhibitors includes small nonnucleoside natural compounds like EGCG (epigallocatechin-3-gallate) and curcumin; however, it has been suggested that the observed inhibition of DNMT1 might be affected by the free radicals formed during degradation of EGCG rather than its direct interaction with enzyme. Another compound phthalimide RG108 functions as a competitive inhibitor and binds directly to the DNMT1 active site. A broad application of RG108 is however limited due to its high hydrophobicity. The third group of DNMT1 inhibitors consists of SAM (S-adenosyl-L-methionine) analogs lacking the methyl group. One of them, sinefungin, increases the rate of $\mathrm{m}^{5} \mathrm{C}$ deamination to thymine. Finally, the fourth group of inhibitors consists of procaine and its derivative procainamide. By binding to the CpG-rich sequences these compounds induce a spatial hindrance preventing the DNA methylation.

Despite significant progress in the research of novel cancer treatment methods based on the regulation of epigenetic processes, search for effective and non-toxic small-molecular inhibitors of DNA methylation is continued. Compounds described till date as potential DNA methyltransferase inhibitors were found to be of poor efficacy in cell line studies. Therefore, effective modulators of DNA methylation that could be used in prevention and treatment of diseases associated with DNA methylation level disorders are required.

The aim of this study was to prepare new cytosine derivatives having a big potential to inhibit DNA methyltransferase activity and methods of their synthesis.

\section{Main patent claims}

A method of preparation of an analog of cytosine or a physiologically acceptable salt or a prodrug, selected 
from the group consisting of $1, \mathrm{~N} 4,5$, and 6 -substituted derivatives of cytosine, 5-azacytosine, or 5,6-dihydrocytosine, where the analog is described with the chemical formulas:<smiles>[R7]c1[Y]([12PH])c(NCC)nc(=O)n1[Z7]</smiles><smiles>[R1]N1C(=O)N=C(NCC)[Y]([R])([H])C1([R])[2H]</smiles>

where R1 is H, R4, 2 -deoxyribosyl, R4 is alkyl or aryl, $\mathrm{X}$ is $\mathrm{N}$ or $\mathrm{C}$; if $\mathrm{X}$ in formula $\mathrm{I}$ is $\mathrm{N}$, then $\mathrm{R} 5$ is no substituent and if $\mathrm{X}$ in formula I and/or II is C or if X in formula II is $\mathrm{N}$, then $\mathrm{R} 5$ and $\mathrm{R} 6$ are independently alkyl, aryl, hydroxyalkyl, aminoalkyl, hydroxyl, carboxyl, amino group, alkoxyl, aryloxyl, aminoalkyl, aminoaryl, thio group, sulfonyl, sulfinyl, or halogen.

The physiologically acceptable salt is a sodium, a calcium, a potassium, or an ammonium salt.

The usage of the analog of cytosine or a physiologically acceptable salt in the treatment of diseases associated with DNA methylation disorders, in particular, the disturbances of DNA methyltransferase 1 activity, so as to inhibit DNA methylation within the cells. 